\title{
La clase de Educación Física a través de la modalidad virtual en el contexto ecuatoriano
}

\section{The Physical Education class through the virtual modality}

1 Segundo Víctor Medina Paredes

iD https://orcid.org/0000-0001-7227-3882

Universidad Técnica de Ambato, Carrera de Pedagogía de la Actividad Física y Deporte, Tungurahua, Ecuador

sv.medina@uta.edu.ec

2 Lenin Esteban Loaiza Dávila

Universidad Técnica de Ambato, Carrera de Pedagogía de la Actividad Física y Deporte, Tungurahua, Ecuador e.loaiza@uta.edu.ec

3 James Santiago Rodríguez Robalino

Universidad Técnica de Ambato, Carrera de Pedagogía de la Actividad Física y Deporte, Tungurahua, Ecuador jamessantiagorodriguezrobalino@gmail.com

4 Patricio Gustavo Ortiz Ortiz

Universidad Técnica de Ambato, Carrera de Pedagogía de la Actividad Física y Deporte, Tungurahua, Ecuador,

patriciogortizo@uta.edu.ec

Artículo de Investigación Científica y Tecnológica

Enviado: 24/12/2021

Revisado: 29/12/2021

Aceptado: $12 / 01 / 2022$

Publicado:08/03/2023

DOI: https://doi.org/10.33262/concienciadigital.v6i1.4.2022

Cítese:

Medina Paredes, S. V., Loaiza Dávila , L. E., Rodríguez Robalino, J. S., \& Ortiz Ortiz, P. G. (2023). La clase de Educación Física a través de la modalidad virtual en el contexto ecuatoriano.

ConcienciaDigital,

6(1.4),

685-700.

https://doi.org/10.33262/concienciadigital.v6i1.4.2022

CONCIENCIA DIGITAL, es una Revista Multidisciplinar, Trimestral, que se publicará en soporte electrónico tiene como misión contribuir a la formación de profesionales competentes con visión humanística y crítica que sean capaces de exponer sus resultados investigativos y científicos en la misma medida que se promueva mediante su intervención cambios positivos en la sociedad. https://concienciadigital.org

La revista es editada por la Editorial Ciencia Digital (Editorial de prestigio registrada en la Cámara

Ecuatoriana de Libro con No de Afiliación 663) www.celibro.org.ec 
Palabras claves: educación física, modalidad virtual, cumplimiento, aplicación.
Keywords: physical education, virtual modality, compliance, implementation.

\section{Resumen}

Introducción. Por efectos de la nueva realidad, producto de la pandemia que vive la humanidad, se han dado transformaciones en todo ámbito, uno de ellos es el del proceso de enseñanza aprendizaje. Objetivo. La presente investigación se focaliza en determinar el cumplimiento de los componentes de la clase de Educación Física y la aplicación de instrumentos didácticos en el proceso de evaluación a través de la modalidad virtual. Metodología. Estudio de enfoque cuantitativo, de tipo no experimental, descriptivo, transversal; con 68 docentes, como muestra de estudio, pertenecientes al Colegio de Profesionales de Cultura Física de Tungurahua. Se empleó la técnica de la encuesta y como instrumento, un cuestionario estructurado, el cual se diseñó y valido por métodos estadísticos y por criterio de expertos en el área de la Educación Física, estructurado para obtener información en base a 5 dimensiones relacionadas al cumplimiento y a la aplicación de componentes de la clase de Educación Física, a través de la modalidad virtual. Resultados. El análisis de las respuestas por dimensión permitió evidenciar que el $51.1 \%$ de los docentes se encontraba en un nivel alto de cumplimiento de los componentes de la clase y el $54.4 \%$ se encontraba en un nivel medio de los procesos dentro de la clase. Conclusiones. Se concluye que, en base a los resultados estadísticos, los docentes poseen un nivel alto de cumplimiento y un nivel medio de aplicación de los componentes de la clase de educación física en la modalidad virtual. Estos resultados permiten plantear alternativas válidas de solución, sobre la base de la realidad analizada.

Abstract

Introduction. Because of the new reality, product of the pandemic that humanity is living, there have been transformations in all areas, one of them is the teaching-learning process. Objective. The present research focuses on determining the compliance of the components of the Physical Education class and the application of didactic instruments in the evaluation process through the virtual modality. Methodology. It responds to a quantitative, basic, nonexperimental approach; 68 teachers participated as a study sample, belonging to the College of Physical Culture Professionals of Tungurahua. The survey technique was used and as an instrument, 
a structured questionnaire, which was designed and validated by statistical methods and by criteria of experts in the area of Physical Education, structured to obtain information based on 5 dimensions related to the fulfillment and application of components of the Physical Education class, through the virtual modality. Results. The analysis of the responses by dimension showed that $51.1 \%$ of the teachers were at a high level of compliance with the components of the class and $54.4 \%$ were at a medium level of the processes within the class. Conclusions. It is concluded that, based on the statistical results, teachers have a high level of compliance and a medium level of application of the components of the physical education class in the virtual modality. These results allow proposing valid solution alternatives, based on the analyzed reality.

\section{Introducción}

El mundo se ha enfrentado a una nueva realidad a partir de finales de 2019 y durante los años 2020 y 2021 por la presencia de COVID - 19 que trastocó lo que se consideraba normal y la humanidad tuvo que adaptarse a una nueva realidad; la educación no ha escapado a esa situación; de forma repentina, la forma de impartir clases imperante pasó a ser la modalidad virtual; el empleo de internet se ha convertido en la herramienta principal y todos los actores de los diferentes niveles educativos se ven obligados a adaptarse a la virtualidad.

Puntualmente, es el caso de la enseñanza virtual de la Asignatura de Educación Física a la que se refiere la presente investigación, ya que también ha sido motivo de novedad, innovación y puesta en práctica de estrategias virtuales para el tratamiento de esta. Estudios como el de Guarnizo y Talero (2021) señalan que la intencionalidad didáctica de los docentes en la mediación de aplicaciones Web 2.0, sobre todo al momento de relacionar el contenido digital desde una mirada pedagógica que se relacione directamente con las prácticas de enseñanza pretenden las prácticas de enseñanza enmarcadas en los procesos de construcción del conocimiento que realizan los estudiantes ante una tarea motriz. Así mismo, se hace necesario considerar que la integración de las TIC a nivel educativo como recurso didáctico permite la adquisición de nuevos aprendizajes (Díaz-Maroto \& Martínez, 2015). Estos recursos han generado en el currículo de la educación física desafíos didácticos, que han permitido organizar competencias docentes en ambientes e-learnig y b-learnig. 
De igual manera, se debe resaltar que, a partir del confinamiento, los docentes de educación física han empleado estrategias en formato multimedia, así, al hablar de este tipo de formato se hace referencia específicamente a la implementación de las nuevas tecnologías de la información y la comunicación, orientadas al mejoramiento de las prácticas de enseñanza de los docentes, como son la educación a distancia (e-learnig) o la educación semi presencial, conocida como educación b-learnig.

Según Cañabate et al. (2018), el aprendizaje debe partir de innovaciones que se den en la clase de educación física, de manera continua, a través de la utilización de recursos TIC por parte del profesorado en el desarrollo de las clases.

En virtud de esas circunstancias, originadas por el confinamiento, se puede discernir que el internet, las redes sociales, los sitios web como YouTube, las plataformas de video como Zomm, Meet y Teams, los teléfonos inteligentes, las Apps y los dispositivos vestibles, llamados en inglés Weberables, permiten el desarrollo de competencias tecnológicas en los estudiantes, a través de la utilización de las TIC en los contenidos curriculares de la clase de educación física.

Cabe destacar el uso de la tecnología móvil en la clase de educación física, pues a través de teléfonos inteligentes los docentes ponen en funcionamiento en clase Apps conocidas como aplicaciones móviles para el desarrollo, algunas veces de trabajo de acondicionamiento físico sin importar si el sistema operativo con que cuente el dispositivo móvil es (iOS o Android), en definitiva, la educación Mobile Learning, es un complemento directo de la educación e-learning y b-learning, es por esto por lo que en el párrafo anterior se menciona la tecnología Weberable en la clase de educación física, pues este tipo de tecnología es el complemento de las aplicaciones móviles que son un elemento directo de las pedagogías disruptivas en la educación física.

Otro estudio, efectuado por Sánchez et al. (2020) señalan que el confinamiento social provocado por el coronavirus, y las medidas del Gobierno Nacional tomadas para precautelar la salud e integridad de los estudiantes, ha generado cambios en los estilos de enseñanza de la Educación Física de una modalidad presencial a una modalidad virtual, ante esta circunstancia los docentes se ven en la necesidad de utilizar los recursos tecnológicos para continuar con el proceso de enseñanza-aprendizaje dirigido a todos los niveles de educación, donde la motivación del docente está promoviendo mejorar significativas en el aspecto pedagógico hacia la construcción de valores afectivos y cognitivos en la educación física (Bonilla et al., 2020). Por lo que es de vital importancia determinar los estilos de enseñanza y recursos digitales que mayor beneficio brinden a la educación física online, manteniendo el objetivo propuesto por el Ministerio de Educación es "que el estudiante alcance su máximo desarrollo de sus capacidades" (p.13), con la misión de "incorporar la actividad física culturalmente significativa en la 
formación integral del ciudadano, para que su práctica habitual, saludable y responsable contribuya a su realización individual y colectiva en el marco del buen vivir" (p.41).

Es necesario analizar los estilos de enseñanza porque ayudan a mejorar las experiencias educativas, los autores Navarro et al. (2010) afirman que el estilo de enseñanza es la "forma de llevar la clase o enseñar" y "se manifiesta en la interacción alumno-profesor" (p.22). Donde el docente debe dominar varios estilos de enseñanza para aplicarlos y combinarlos de acuerdo con las necesidades de los alumnos para guiarlos hacia el aprendizaje, a partir del estilo propio que se sustenta de la formación académica, personal y humana del docente. Se podría hablar de una hibridación de estilos (Navarro et al., 2010), de forma holística que absorba las características más importantes del acto educativo para combinar los estilos de enseñanza con el objeto de dar respuestas a las necesidades de los alumnos, ya que es difícil mantener un solo estilo de enseñanza, sino al contrario se utilizan diversos estilos en el acto educativo como menciona Ruiz et al. (2019) la necesidad de conocer diferentes estilos de enseñanza para aplicarlos en las clases de Educación Física, con el fin de obtener un aprendizaje significativo y en algunos casos funcional para el alumno, manteniendo siempre un estilo de principal y otro de secundario (Navarro et al., 2010).

De acuerdo con los estilos de enseñanza más utilizados en la educación física según Ramírez y Noguera (1999) clasifican en dos grupos:

El reproductivo y productivo, el primero hace referencia a una pedagogía tradicional, individual, participativa y socializadora donde el docente toma la mayor parte de las decisiones, los estilos de enseñanza que forman parte de este grupo son el mando directo y la asignación de tareas, los programas individuales, trabajo por grupos, la enseñanza modulada, la enseñanza programada, la enseñanza recíproca, grupos reducidos, la microenseñanza y estilo cooperativo, por otro lado se encuentra el productivo que involucra un estilo de enseñanza cognitivo y creativo en este grupo se encuentra los estilos de descubrimiento guiado, la resolución de problemas y la sinéctica que favorece la creatividad.

Estos estilos de enseñanza de la Educación Física se pueden argumentar con los trabajos de Hernández \& Infante (2016) que sostienen que los estilos más utilizados son la instrucción o mando directo, desde el punto de vista tradicional y desde el ámbito constructivo, es el cognitivo que abarca los estilos por descubrimiento y resolución de problemas. Sumando a ello la perspectiva motivacional que contribuye al aprendizaje significativo (Ruiz et al., 2019).

En el contexto de la modificación y adaptación de la virtualidad, aparece un punto relevante, como es el de la formación de docentes. Al respecto Romero y Kabusch (2017) sostienen que el proceso de reflexión y construcción de conocimiento llevado a cabo por 
los discentes durante el cursado de sus prácticas docentes es un proceso complejo y multifacético en donde se ponen en juego diferentes destrezas y estrategias que requieren un procesamiento ágil de toda la información que necesita para llevar a cabo su actividad. De igual manera, las intervenciones del docente son permanentes y en un continuo espacio de retroalimentación que sostiene la actividad del discente practicante.

Es innegable, por otro lado, el hecho de que ha existido desde siempre desigualdad en la educación, la virtualidad, por el tema de acceso a herramientas tecnológicas, cobertura de internet son insumos que agravan esa heterogeneidad; el estudio efectuado por Gómez y Escobar (2021) sostienen que en los últimos años se ha incrementado el acceso al sistema educativo; mas no se ha podido superar el tema de la calidad heterogénea del servicio. Como sostiene De Belaúnde et al. (2011), ampliar la cobertura educativa ha conducido a que la población tenga más acceso al servicio educativo; pero no se han generado cambios sustanciales para mejorar la calidad, manteniéndose las desigualdades entre los servicios público y privado; urbano y rural; así como entre la población indígena y no indígena.

La Didáctica de la educación física centra su estudio en las relaciones profesor/alumno que se establecen en un proceso intencional de enseñanza y aprendizaje en torno al movimiento humano como objeto de comunicación. Dicho estudio se encuentra orientado hacia la búsqueda de explicaciones sobre el cómo hacer posible que el alumno desarrolle sus actividades de aprendizaje en aquellas condiciones que faciliten la significatividad y eficacia de tales actividades. Explicaciones que deberán validarse y reconstruirse a través de propuestas concretas de intervención docente orientadas por las finalidades educativas que se persiguen. Así pues, entendiendo el estudio del proceso de enseñanza y aprendizaje desde la doble perspectiva que lleva a su explicación (teoría) y a la intervención (práctica), podemos afirmar que el objeto de la Didáctica de la Educación Física debe centrarse en el binomio "enseñanza y aprendizaje de las manifestaciones del movimiento humano", entendido este no como un simple fenómeno físico sino cargado de intencionalidad y significado para el ser que se mueve.

Para que estos estilos de enseñanza trasciendan a la modalidad virtual es necesario involucrar las tecnologías de la información y comunicación con las tecnologías del aprendizaje y conocimiento para posibilitar el proceso de enseñanza a un aprendizaje $e$ Learning, respaldados en Barahona (2012) quien derriba la discrepancia entre la Educación Física y la tecnología y a su vez describe las ventajas que brinda su conexión; entre las cuales desarrolla la colaboración y trabajo en equipo, permite crear autonomía e iniciativa personal por aprender, utilizar diferentes espacios a comodidad del estudiante, facilita la transmisión y búsqueda de contenidos e información, permite tener una comunicación permanente a pesar de la distancia.

En el libro la didáctica de la educación física en educación infantil y primaria en las propuestas metodológicas para una educación física del siglo XXI describe la importancia 
y recomienda herramientas digitales para planificar de forma sistemática donde sobresale la organización, la comunicación, el desarrollo de sesiones y la evaluación que llevaran al éxito el proceso educativo (Carriedo y Cecchini, 2019). Por otro lado, Prieto (2020) asegura que la educación online es totalmente viable hacia una educación a distancia donde el estudiante puede acceder a video llamadas o reuniones virtuales, contenidos y tareas. Por lo que propone algunas herramientas y recursos digitales que pueden ser utilizados en las clases de Educación Física como; la herramienta 2.0, aprendizaje basado en juegos-gamificación, las aplicaciones móviles de actividad física en IOS/ANDROID y los blogs y recursos electrónicos.

Como un método innovador y que va anclado con la tecnología y los recursos digitales educativos es necesario hablar de la gamificación, considerado como una metodología atractiva para el educando que se fundamenta en una historia o narrativa para alcanzar los aprendizajes requeridos (Aguilar et al., 2019). De igual forma Foncubierta y Rodríguez (2014) definen a la gamificación como un proceso de aprendizaje que se vale de las funciones y elementos del juego con fines pedagógicos, que usa neurotransmisores como la dopamina y el placer para motivar el aprendizaje (Prieto, 2020), esto contribuiría en el aprendizaje de los alumnos en este aislamiento social relacionando los videos-juegos hacia el desarrollo de habilidades y destrezas motoras.

En base a la problemática estudiada y la revisión bibliográfica de sustento, se plantea como objetivo de la investigación determinar el cumplimiento de los componentes de la clase de Educación Física y la aplicación de instrumentos didácticos en el proceso de evaluación a través de la modalidad virtual.

\section{Metodología}

La investigación se desarrolla en base a un enfoque cuantitativo, sustentado desde el análisis de los datos numéricos obtenidos con la investigación de campo. Aplica una investigación bibliográfica, que permitió realizar un análisis de varias fuentes escritas sobre la problemática en estudio y de campo al obtener datos directamente de los sujetos involucrados en el estudio. Por su alcance responde a un estudio correlacional involucrando a las variables de nivel de conocimiento y de aplicación de los componentes de la clase de educación física, además se aplicó un corte trasversal, ya que en un determinado tiempo y espacio se aplicó el instrumento diseñado.

La muestra de estudio estuvo conformada por 68 docentes de sistema educativo ecuatoriano de formación media, en especial por docentes del colegio de profesionales de Cultura Física de la provincia de Tungurahua.

Como técnica de investigación que se aplicó la encuesta y como instrumento un cuestionario basado en 48 ítems con respuestas en una escala del Likert de 5 niveles que 
fue validad por criterio de expertos en el área de la educación física y estadísticamente a través de la prueba Alfa de Cronbach obteniendo un coeficiente de 0.938, valor muy cercano al valor 1 que establece que el instrumento construido posee un nivel de confianza muy bueno y puede ser utilizado para la investigación pertinente.

El cuestionario estuvo estructurado por cinco dimensiones, las tres primeras dimensiones desde el ítem 1 a al15 fueron cuestionamientos referentes al cumplimiento de los objetivos del currículo, contenidos de este y a la aplicación de métodos de enseñanza; del ítem 16 al 48 correspondientes a las dos últimas dimensiones se establecieron cuestionamientos relacionados a la aplicación de medios e instrumentos didácticos y a la aplicación de tipos e instrumentos de evaluación de aprendizajes.

El análisis estadístico de los resultados se realizó a través del programa estadístico SPSS con su respectiva versión 25, ejecutando pruebas de distribución frecuencial, porcentual, descriptivos de valores medios, así como la prueba de normalidad de KolmogórovSmirnov que determino la aplicación de la prueba no paramétrica de U de MannWhitney para muestras independientes y por último la prueba de Chi-cuadrado de Pearson para determinar el grado de correlación entre las variables estudiadas.

\section{Resultados}

En primer lugar, se presenta un estudio de caracterización de la muestra de estudio

Tabla 1

Categorización de la muestra de estudio

\begin{tabular}{|c|c|c|c|c|c|c|c|c|}
\hline \multirow{2}{*}{ Sexo } & \multirow{2}{*}{$\mathrm{f}$} & \multirow{2}{*}{$\%$} & \multicolumn{3}{|c|}{ Edad (años) } & \multicolumn{3}{|c|}{ Experiencia (años) } \\
\hline & & & $\mathrm{M}$ & DS & $\mathrm{P}$ & $\mathrm{M}$ & DS & $\mathrm{P}$ \\
\hline Masculino & 55 & 80.9 & 41.95 & 8.648 & & 13.89 & 9.655 & \\
\hline Femenino & 13 & 19.1 & 41.62 & 8.332 & $0.901 *$ & 16.15 & 9.720 & $0.496^{*}$ \\
\hline Total & 68 & 100.0 & 41.88 & 8.528 & & 14.32 & 9.636 & \\
\hline
\end{tabular}

Nota. Análisis de frecuencias (f) y porcentajes (\%); análisis descriptivo de valores medios (M) y desviaciones estándares con un nivel de significación estadística de $\mathrm{P} \geq$ entre grupos.

La tabla 1 muestra la categorización de la muestra de estudio, en donde se observa que existe un mayor porcentaje de docentes del sexo masculino en un $68.8 \%$ más que el grupo de sexo femenino y sin diferencias significativas en un nivel de $\mathrm{P} \geq 0.05$ en relación con las variables de la edad y experiencia de ejercicio profesional. De igual manera se puede observar la distribución del sector en el cual laboran (tabla 2). 


\section{Tabla 2}

Distribución por sector educativo

\begin{tabular}{ccc}
\hline Sector & Frecuencia & Porcentaje (\%) \\
\hline Publica & 47 & 69.1 \\
Privada & 21 & 30.9 \\
\hline Total & 68 & 100.0 \\
\hline
\end{tabular}

Determinando que la mayoría de los docentes se desempeñan en el sector público de educación. La aplicación del instrumento de investigación permitió evidenciar los resultados por dimensiones planteadas.

Dimensión 1. Cumplimiento de objetivos del currículo.

Se analizaron el nivel del cumplimiento de los objetivos generales y específicos del currículo (tabla 3).

\section{Tabla 3}

Resultados de la valoración del cumplimiento de los objetivos del currículo en la muestra de estudio

\begin{tabular}{lcccc}
\hline \multirow{2}{*}{ Opciones de cumplimiento } & \multicolumn{2}{c}{ Objetivos generales } & \multicolumn{2}{c}{ Objetivos específicos } \\
\cline { 2 - 5 } & $\mathrm{f}$ & $\%$ & $\mathrm{f}$ & $\%$ \\
\hline No lo cumplo & 2 & 2.9 & 0 & 0 \\
Casi no lo cumplo & 9 & 13.2 & 13 & 19.1 \\
Lo cumplo regularmente & 17 & 25.0 & 17 & 25.0 \\
Lo cumplo frecuentemente & 17 & 25.0 & 20 & 29.4 \\
Siempre lo cumplo & 23 & 33.8 & 18 & 26.5 \\
\hline Total & 68 & 100.0 & 68 & 100.0 \\
\hline
\end{tabular}

Nota. Distribución de frecuencias (f) y porcentajes (\%) por ítems dimensión 1.

En relación con los objetivos generales el mayor porcentaje de encuestados determino que existe un cumplimiento total y frecuente, en relación con los objetivos específicos en donde el mayor porcentaje determino que frecuentemente cumple.

\section{Dimensión 2. Desarrollo de contenidos del currículo.}

Se analizaron el nivel de cumplimiento de los contenidos basados en los bloques curriculares: Prácticas lúdicas (PL), prácticas gimnasticas (PG), prácticas corporales expresivo-comunicativas (PCEC), prácticas deportivas (PD), relación en las prácticas corporales y la salud (R-PCS) y construcción de la identidad corporal (CIC) establecidas en la tabla 4. 


\section{Tabla 4}

Resultados de la valoración del desarrollo de contenidos del currículo en la muestra de estudio

\begin{tabular}{|c|c|c|c|c|c|c|c|c|c|c|c|c|}
\hline \multirow{2}{*}{$\begin{array}{l}\text { Opciones de } \\
\text { cumplimiento }\end{array}$} & \multicolumn{2}{|c|}{ PL } & \multicolumn{2}{|c|}{ PG } & \multicolumn{2}{|c|}{ PCEC } & \multicolumn{2}{|c|}{ PD } & \multicolumn{2}{|c|}{ R-PCS } & \multicolumn{2}{|c|}{$\mathrm{CIC}$} \\
\hline & $\mathrm{f}$ & $\%$ & $\mathrm{f}$ & $\%$ & $\mathrm{f}$ & $\%$ & $\mathrm{f}$ & $\%$ & $\mathrm{f}$ & $\%$ & $\mathrm{f}$ & $\%$ \\
\hline No lo cumplo & 3 & 4.4 & 7 & 10.3 & 0 & 0 & 4 & 5.9 & 0 & 0 & 2 & 2.9 \\
\hline Casi no lo cumplo & 10 & 14.7 & 13 & 19.1 & 11 & 16.2 & 16 & 23.5 & 8 & 11.8 & 6 & 8.8 \\
\hline $\begin{array}{l}\text { Lo cumplo } \\
\text { regularmente }\end{array}$ & 19 & 27.9 & 20 & 29.4 & 20 & 29.4 & 14 & 20.6 & 19 & 27.9 & 21 & 30.9 \\
\hline $\begin{array}{l}\text { Lo cumplo } \\
\text { frecuentemente }\end{array}$ & 17 & 25.0 & 17 & 25.0 & 14 & 20.6 & 18 & 26.5 & 19 & 27.9 & 22 & 32.4 \\
\hline Siempre lo cumplo & 19 & 27.9 & 11 & 16.2 & 23 & 33.8 & 16 & 23.5 & 22 & 32.4 & 17 & 25.0 \\
\hline Total & 68 & 100 & 68 & 100 & 68 & 100 & 68 & 100 & 68 & 100 & 68 & 100 \\
\hline
\end{tabular}

El análisis respectivo determino que existió un mayor cumplimiento de los contenidos de las prácticas corporales expresivo-comunicativas y de los contenidos del bloque de relación entre las prácticas corporales y la salud.

Dimensión 3. Aplicación de métodos enseñanza.

Se analizaron el nivel de cumplimento de los métodos de enseñanza: Aprendizaje basado en problemas (ABP), aprendizaje basado en proyectos (ABPRO), flipped clasroom - aula invertida $(\mathrm{AI})$, aprendizaje basado en el juego (ABJ), aprendizaje cooperativo (AC), aprendizaje basado en el pensamiento (ABPENS) y aprendizaje basado en el dialogo (ABD) establecidos en la tabla 5.

\section{Tabla 5}

Resultados de la valoración de la aplicación de métodos de enseñanza en la muestra de estudio

\begin{tabular}{|c|c|c|c|c|c|c|c|c|c|c|c|c|c|c|}
\hline \multirow{2}{*}{$\begin{array}{c}\text { Opciones de } \\
\text { cumplimiento }\end{array}$} & \multicolumn{2}{|c|}{$\mathrm{ABP}$} & \multicolumn{2}{|c|}{ ABPRO } & \multicolumn{2}{|c|}{$\mathrm{AI}$} & \multicolumn{2}{|c|}{$\mathrm{ABJ}$} & \multicolumn{2}{|c|}{$\mathrm{AC}$} & \multicolumn{2}{|c|}{ ABPENS } & \multicolumn{2}{|c|}{$\mathrm{ABD}$} \\
\hline & $\mathrm{f}$ & $\%$ & $\mathrm{f}$ & $\%$ & $\mathrm{f}$ & $\%$ & 1 & $\%$ & $\mathrm{f}$ & $\%$ & $\mathrm{f}$ & $\%$ & $\mathrm{f}$ & $\%$ \\
\hline No lo cumplo & 1 & 1.5 & 3 & 4.4 & 3 & 4.4 & 0 & 0 & 5 & 7.4 & 4 & 5.9 & 0 & 0 \\
\hline Casi no lo cumplo & 12 & 17.6 & 4 & 5.9 & 10 & 14.7 & 8 & 11.8 & 7 & 10.3 & 2 & 2.9 & 6 & 8.8 \\
\hline $\begin{array}{l}\text { Lo cumplo } \\
\text { regularmente }\end{array}$ & 17 & 25.0 & 17 & 25.0 & 22 & 32.4 & 18 & 26.5 & 14 & 20.6 & 20 & 29.4 & 14 & 20.6 \\
\hline $\begin{array}{l}\text { Lo cumplo } \\
\text { frecuentemente }\end{array}$ & 20 & 29.4 & 27 & 39.7 & 27 & 39.7 & 21 & 30.9 & 31 & 45.6 & 32 & 47.1 & 29 & 42.6 \\
\hline $\begin{array}{l}\text { Siempre lo } \\
\text { cumplo }\end{array}$ & 18 & 26.5 & 17 & 25.0 & 6 & 8.8 & 21 & 30.9 & 11 & 16.2 & 10 & 14.7 & 19 & 27.9 \\
\hline Total & 68 & 100 & 68 & 100 & 68 & 100 & 68 & 100 & 68 & 100 & 68 & 100 & 68 & 100 \\
\hline
\end{tabular}

El análisis determino que el aprendizaje basado en el juego (ABJ) fue el método de enseñanza más utilizado por los docentes en el proceso virtual de educación, seguido por una utilización frecuente de aprendizaje cooperativo (AC), aprendizaje basado en el pensamiento (ABPENS) y aprendizaje basado en el dialogo (ABD). 
Dimensión 4. Aplicación de medios e instrumentos didácticos

Se analizaron la aplicación de los diferente plataformas educativas, programas informáticos y redes sociales de mayor accesibilidad por parte de los docentes (tabla 6).

\section{Tabla 6}

Resultados de la valoración de aplicación de medios e instrumentos didácticos por la muestra de estudio

\begin{tabular}{|c|c|c|c|c|c|c|c|c|c|c|c|c|}
\hline \multirow{2}{*}{$\begin{array}{l}\text { Opciones de } \\
\text { cumplimiento }\end{array}$} & \multicolumn{2}{|c|}{$\begin{array}{l}\text { No lo } \\
\text { aplico }\end{array}$} & \multicolumn{2}{|c|}{$\begin{array}{l}\text { Casi no lo } \\
\text { aplico }\end{array}$} & \multicolumn{2}{|c|}{$\begin{array}{l}\text { Lo aplico } \\
\text { regularmente }\end{array}$} & \multicolumn{2}{|c|}{$\begin{array}{c}\text { Lo aplico } \\
\text { frecuentemente }\end{array}$} & \multicolumn{2}{|c|}{$\begin{array}{l}\text { Siempre lo } \\
\text { aplico }\end{array}$} & \multicolumn{2}{|c|}{ Total } \\
\hline & $\mathrm{f}$ & $\%$ & $\mathrm{f}$ & $\%$ & $\mathrm{f}$ & $\%$ & $\mathrm{f}$ & $\%$ & $\mathrm{f}$ & $\%$ & $\mathrm{f}$ & $\%$ \\
\hline RUNACHAY & 29 & 42.6 & 11 & 16.2 & 8 & 11.8 & 15 & 22.1 & 5 & 7.4 & 68 & 100 \\
\hline $\begin{array}{l}\text { MICROSOFT } \\
\text { TEAMS }\end{array}$ & 17 & 25.0 & 0 & 0 & 6 & 8.8 & 17 & 25.0 & 28 & 41.2 & 68 & 100 \\
\hline ZOOM & 15 & 22.1 & 4 & 5.9 & 5 & 7.4 & 13 & 19.1 & 31 & 45.6 & 68 & 100 \\
\hline GOOGLE MEET & 33 & 48.5 & 11 & 16.2 & 3 & 4.4 & 10 & 14.7 & 11 & 16.2 & 68 & 100 \\
\hline WORD & 8 & 11.8 & 3 & 4.4 & 10 & 14.7 & 22 & 32.4 & 25 & 36.8 & 68 & 100 \\
\hline EXCEL & 12 & 17.6 & 5 & 7.4 & 15 & 22.1 & 14 & 20.6 & 22 & 32.4 & 68 & 100 \\
\hline POWER POINT & 2 & 2.9 & 4 & 5.9 & 8 & 11.8 & 24 & 35.3 & 30 & 44.1 & 68 & 100 \\
\hline PDF & 2 & 2.9 & 11 & 16.2 & 8 & 11.8 & 17 & 25.0 & 30 & 44.1 & 68 & 100 \\
\hline YOUTUBE & 2 & 2.9 & 4 & 5.9 & 7 & 10.3 & 24 & 35.3 & 31 & 45.6 & 68 & 100 \\
\hline BLOGS & 19 & 27.9 & 16 & 23.5 & 9 & 13.2 & 14 & 20.6 & 10 & 14.7 & 68 & 100 \\
\hline PAGINAS WEB & 5 & 7.4 & 4 & 5.9 & 12 & 17.6 & 27 & 39.7 & 20 & 29.4 & 68 & 100 \\
\hline WHATSAPP & 1 & 1.5 & 7 & 10.3 & 6 & 8.8 & 15 & 22.1 & 39 & 57.4 & 68 & 100 \\
\hline MESSENGER & 25 & 36.8 & 16 & 23.5 & 9 & 13.2 & 14 & 20.6 & 4 & 5.9 & 68 & 100 \\
\hline TELEGRAM & 38 & 55.9 & 14 & 20.6 & 3 & 4.4 & 9 & 13.2 & 4 & 5.9 & 68 & 100 \\
\hline FACEBOOK & 39 & 57.4 & 10 & 14.7 & 11 & 16.2 & 5 & 7.4 & 3 & 4.4 & 68 & 100 \\
\hline TWITER & 51 & 75.0 & 4 & 5.9 & 3 & 4.4 & 8 & 11.8 & 2 & 2.9 & 68 & 100 \\
\hline INSTAGRAM & 51 & 75.0 & 4 & 5.9 & 5 & 7.4 & 6 & 8.8 & 2 & 2.9 & 68 & 100 \\
\hline LINKEDLN & 50 & 73.5 & 5 & 7.4 & 7 & 10.3 & 4 & 5.9 & 2 & 2.9 & 68 & 100 \\
\hline $\begin{array}{l}\text { CORREOS } \\
\text { ELECTRÓNICOS }\end{array}$ & 8 & 11.8 & 12 & 17.6 & 7 & 10.3 & 19 & 27.9 & 22 & 32.4 & 68 & 100 \\
\hline
\end{tabular}

El análisis determino que los docentes desarrollaban su proceso de enseñanza a través del envío de recurso y tareas por WHATSAPP, desarrollando videos ilustrativos y colgándolos en YOUTUBE, los contenidos se enviaban en formatos POWER POINT y PDF y las plataformas de conferencias síncronas fueron ZOOM y MICROSOFT TEAMS.

Dimensión 6. Aplicación de tipos e instrumentos de evaluación de aprendizajes

Se analizaron el nivel de aplicación de los diferentes tipos de evaluación, así como los instrumentos utilizados por los docentes para el desarrollo del proceso evaluativo. 


\section{Tabla 7}

Resultados de la valoración de aplicación de los tipos e instrumentos de evaluación de aprendizajes por la muestra de estudio

\begin{tabular}{|c|c|c|c|c|c|c|c|c|c|c|c|c|}
\hline \multirow{2}{*}{$\begin{array}{l}\text { Opciones de } \\
\text { cumplimiento }\end{array}$} & \multicolumn{2}{|c|}{$\begin{array}{l}\text { No lo } \\
\text { aplico }\end{array}$} & \multicolumn{2}{|c|}{$\begin{array}{l}\text { Casi no lo } \\
\text { aplico }\end{array}$} & \multicolumn{2}{|c|}{$\begin{array}{l}\text { Lo aplico } \\
\text { regularmente }\end{array}$} & \multicolumn{2}{|c|}{$\begin{array}{c}\text { Lo aplico } \\
\text { frecuentemente }\end{array}$} & \multicolumn{2}{|c|}{$\begin{array}{l}\text { Siempre lo } \\
\text { aplico }\end{array}$} & \multicolumn{2}{|c|}{ Total } \\
\hline & $\mathrm{f}$ & $\%$ & $\mathrm{f}$ & $\%$ & $\mathrm{f}$ & $\%$ & $\mathrm{f}$ & $\%$ & $\mathrm{f}$ & $\%$ & f & $\%$ \\
\hline Heteroevaluación & 9 & 13.2 & 14 & 20.6 & 15 & 22.1 & 18 & 26.5 & 12 & 17.6 & 68 & 100 \\
\hline Coevaluación & 5 & 7.4 & 10 & 14.7 & 16 & 23.5 & 21 & 30.9 & 16 & 23.5 & 68 & 100 \\
\hline Autoevaluación & 1 & 1.5 & 4 & 5.9 & 19 & 27.9 & 26 & 38.2 & 18 & 26.5 & 68 & 100 \\
\hline $\begin{array}{l}\text { Observación de } \\
\text { actitudes }\end{array}$ & 0 & 0 & 0 & 0 & 20 & 29.4 & 23 & 33.8 & 25 & 36.8 & 68 & 100 \\
\hline Registro anecdótico & $\begin{array}{l}1 \\
3\end{array}$ & 19.1 & 9 & 13.2 & 16 & 23.5 & 21 & 30.9 & 9 & 13.2 & 68 & 100 \\
\hline Escala de actitudes & 7 & 10.3 & 15 & 22.1 & 16 & 23.5 & 17 & 25.0 & 13 & 19.1 & 68 & 100 \\
\hline $\begin{array}{l}\text { Cuaderno alumnados } \\
\text { de actitudes }\end{array}$ & $\begin{array}{l}1 \\
3\end{array}$ & 19.1 & 9 & 13.2 & 22 & 32.4 & 12 & 17.6 & 12 & 17.6 & 68 & 100 \\
\hline $\begin{array}{l}\text { Cuaderno alumnado } \\
\text { de conceptos }\end{array}$ & $\begin{array}{l}1 \\
3\end{array}$ & 19.1 & 16 & 23.5 & 22 & 32.4 & 11 & 16.2 & 6 & 8.8 & 68 & 100 \\
\hline $\begin{array}{l}\text { Examen de } \\
\text { desarrollo }\end{array}$ & $\begin{array}{l}1 \\
4\end{array}$ & 20.6 & 16 & 23.5 & 12 & 17.6 & 21 & 30.9 & 5 & 7.4 & 68 & 100 \\
\hline Examen tipo test & $\begin{array}{l}1 \\
1\end{array}$ & 16.2 & 10 & 14.7 & 16 & 23.5 & 23 & 33.8 & 8 & 11.8 & 68 & 100 \\
\hline $\begin{array}{l}\text { Observación de } \\
\text { procedimientos }\end{array}$ & 4 & 5.9 & 0 & 0 & 22 & 32.4 & 26 & 38.2 & 16 & 23.5 & 68 & 100 \\
\hline Trabajo práctico & 6 & 8.8 & 4 & 5.9 & 8 & 11.8 & 35 & 51.5 & 15 & 22.1 & 68 & 100 \\
\hline $\begin{array}{l}\text { Test de ejecución } \\
\text { motriz }\end{array}$ & 8 & 11.8 & 7 & 10.3 & 20 & 29.4 & 18 & 26.5 & 15 & 22.1 & 68 & 100 \\
\hline $\begin{array}{l}\text { Test de condición } \\
\text { física }\end{array}$ & 8 & 11.8 & 12 & 17.6 & 20 & 29.4 & 14 & 20.6 & 14 & 20.6 & 68 & 100 \\
\hline
\end{tabular}

El análisis determino que los procesos evaluativos más aplicados por los docentes fueron la observación de actitudes, la autoevaluación, la coevaluación y la observación de procedimientos realizados por el alumno.

El análisis global y sumatoria del puntaje obtenido por cada dimensión, permitió establecer el nivel de cumplimiento y aplicación de los componentes de la clase de educación física bajo la modalidad virtual, para cual se establecieron baremos específicos creados para el instrumento, los cuales se basaron en la relación entre el promedio general y la dispersión positiva o negativa de su desviación estándar (tabla 8)

\section{Tabla 8}

Baremos específicos de los niveles de cumplimiento y aplicación

\begin{tabular}{ccc}
\hline Nivel & Cumplimiento & Aplicación \\
\hline ALTO & $54-75$ & $161-240$ \\
MEDIO & $37-53$ & $96-160$ \\
BAJO & $15-36$ & $16-95$ \\
\hline
\end{tabular}


La contabilización de los diferentes puntajes obtenidos permitió observar los siguientes resultados (tabla 9).

\section{Tabla 9}

Niveles de cumplimiento de los componentes de la clase de educación física y la aplicación de instrumentos didácticos y del proceso de evaluación a través de la modalidad virtual por la muestra de estudio

\begin{tabular}{cccccc}
\hline Variables de estudio & $\mathrm{n}$ & Mín & Máx & M & DS \\
\hline Cumplimiento de objetivos del currículo & & 3.00 & 10.00 & 7.37 & 2.19 \\
Desarrollo de contenidos del currículo & 68 & 10.00 & 30.00 & 21.34 & 6.25 \\
$\quad$ Aplicación de métodos enseñanza & & 12.00 & 35.00 & 25.66 & 5.83 \\
\hline Total cumplimiento & 68 & 27.00 & 75.00 & 54.45 & 13.41 \\
\hline $\begin{array}{c}\text { Medios e instrumentos didácticos } \\
\text { Aplicación de tipos e instrumentos de } \\
\text { evaluación de aprendizajes }\end{array}$ & 68 & 32.00 & 95.00 & 55.99 & 14.94 \\
$\quad$ Total aplicación & 68 & 63.00 & 165.00 & 102.46 & 25.80 \\
\hline
\end{tabular}

La aplicación de los respectivos baremos por variable analizada permitió observar los siguientes resultados (tabla 10).

\section{Tabla 10}

Niveles de distribución frecuencial y porcentual de cumplimiento y aplicación de los componentes de la clase de educación física en la modalidad virtual por la muestra de estudio

\begin{tabular}{ccccc}
\hline \multirow{2}{*}{ Niveles } & \multicolumn{2}{c}{ Cumplimiento } & \multicolumn{2}{c}{ Aplicación } \\
\cline { 2 - 5 } & $\mathrm{f}$ & $\%$ & $\mathrm{f}$ & $\%$ \\
\hline Bajo & 7 & 10.3 & 29 & 42.6 \\
Medio & 26 & 38.2 & 37 & 54.4 \\
Alto & 35 & 51.5 & 2 & 2.9 \\
\hline Total & 68 & 100.0 & 68 & 100.0
\end{tabular}

El análisis de correlación a través de la aplicación de la prueba Chi-cuadrado de Pearson en primer lugar permitió observar cómo en relación a los diferentes niveles de conocimiento, se distribuyeron los niveles de aplicación de los componentes, y estadísticamente se observó que existen diferencias significativas en un nivel de $\mathrm{P}<0.05$, determina que existe una relación lineal entre las dos variables estudiadas, es decir que del nivel de cumplimiento depende el nivel de aplicación por parte de los docente.

\section{Conclusiones}

- Se concluye, en base a los resultados estadísticos, que los docentes poseen un nivel alto de cumplimiento y un nivel medio de aplicación de los componentes de la clase 
de educación física en la modalidad virtual. Estos resultados permiten plantear alternativas válidas de solución, sobre la base de la realidad analizada.

- La investigación permite evidenciar, mediante un análisis descriptivo la importancia de la educación virtual en un ámbito tan trascendental de la formación de las personas como lo es la Educación Física, se aborda el empleo de estrategias, métodos, procedimientos, técnicas de evaluación que complementan el desarrollo de la asignatura en una modalidad necesaria y útil en los actuales momentos.

- Hay una relación recíproca, necesaria y vital entre la planificación, ejecución y evaluación del proceso de enseñanza - aprendizaje en todos los niveles del proceso. El caso de la educación física, a través de la modalidad virtual, no escapa a esa realidad y, se enfatiza con el hecho de involucrar a los actores en niveles óptimos de cumplimiento de objetivos y adquisición de aprendizajes significativos.

\section{Referencias bibliográficas}

Aguilar Villalobos, P. M., Briones Sánchez, J. R., \& Córdova López, R. N. (2019). La gamificación como estrategia metodológica y la gestión de herramientas ofimáticas como logro de aprendizaje en los estudiantes de primer ciclo, en la asignatura de Fundamentos de Informática de la Facultad de Administración y Negocios de una universidad privada de Lima, en 2018 II.

Barahona, J. D. (2012). La enseñanza de la Educación Física implementada con TIC. Educación física y deporte, 31(2), 1056-1056.

Bonilla, J. A. M., Benítez, M. C., \& Galak, E. L. (2020). Apoyo motivacional docente y necesidades psicológicas básicas en la Educación Física: una revisión sistemática. Ciencia Digital, 4(1.1.), 5-20.

Cañabate, D., Colomer, J., \& Olivera, J. (2018). Movement: A Language for Growing. Apunts: Educació Física i Esports, (134).

Carriedo, A., \& Cecchini, J. A. (2019). ¿Cómo aumentar la actividad física diaria dentro del horario escolar? ejemplo de un proyecto interdisciplinar entre educación física y matemáticas. Journal of Sport \& Health Research, 11.

De Belaúnde, C., Trivelli, C., \& Israel, C. (2011). Inclusión a través de la educación de posgrado: el Programa Internacional de Becas de la Fundación Ford.

Díaz-Maroto, I. T., \& Martínez, A. C. (2015). Las TIC y las necesidades específicas de apoyo educativo: análisis de las competencias TIC en los docentes. RIED. Revista Iberoamericana de Educación a Distancia, 18(2), 355-383. 
Foncubierta, J. M., \& Rodríguez, C. (2014). Didáctica de la gamificación en la clase de español. Madrid: Edinumen. Recuperado de: https://www. edinumen. es/spanish_challenge/gamificacion_didactica. pdf (consultado el 14 de noviembre de 2018).

Gómez, I., \& Escobar, F. (2021). Educación virtual en tiempos de pandemia: Incremento de la desigualdad social. Scielo, 1 - 13.

Guarnizo, N., \& Talero, E. (2021). El currículo de la educación física en modalidad elearnig y b-learning durante el confinamiento. Acción Motriz, 57 - 65.

Hernández R., \& Infante M. (2016). El método de enseñanza-aprendizaje de trabajo independiente en la clase encuentro: Recomendaciones didácticas. Revista de Pedagogía, 215-231. Obtenido de https://www.redalyc.org/pdf/659/65950543011.pdf

Navarro Reyes Y., Pereira Burgos M., Pereira de Homes L., \& Fonseca Cascioli N. (2010). Una mirada a la planificación estratégica curricular. Telos, 202-216. Obtenido de https://www.redalyc.org/articulo.oa?id=99315569006

Prieto Andreu, J. M. (2020). Recursos digitales desde la educación fisica. La tecnología educativa hoy, 102.

Ramírez, J. V., \& Noguera, M. Á. D. (1999). La programación e intervención didáctica en el deporte escolar (II). Aportaciones de los diferentes estilos de enseñanza. Apunts. Educación física y deportes, 2(56), 17-24.

Romero, C., \& Kabusch, A. (2017). Implementación de entornos virtuales de aprendizaje en el desarrollo de la práctica docente en Educación Física. Memoria Académica, $1-16$.

Ruiz Heredia C., Lara Sánchez A., López Gallego F., Cachón Zagalaz J., \& Valdivia Moral P. (2019). Análisis del tiempo de clase en EF y propuestas para su optimización. Retos: nuevas tendencias en educación física, deporte y recreación, 126-129. Obtenido de https://dialnet.unirioja.es/servlet/articulo?codigo=6761654

Sánchez, E., Ávila, C., \& García, D. (2020). El proceso de enseñanza-aprendizaje de la Educación Física en época de pandemia. Polo del Conocimiento, 455 - 467.

\section{LCiencia}


El artículo que se publica es de exclusiva responsabilidad de los autores y no necesariamente reflejan el pensamiento de la Revista Conciencia Digital.

\section{Ciencia \\ LDigital}

El artículo queda en propiedad de la revista y, por tanto, su publicación parcial y/o total en otro medio tiene que ser autorizado por el director de la Revista Conciencia Digital.
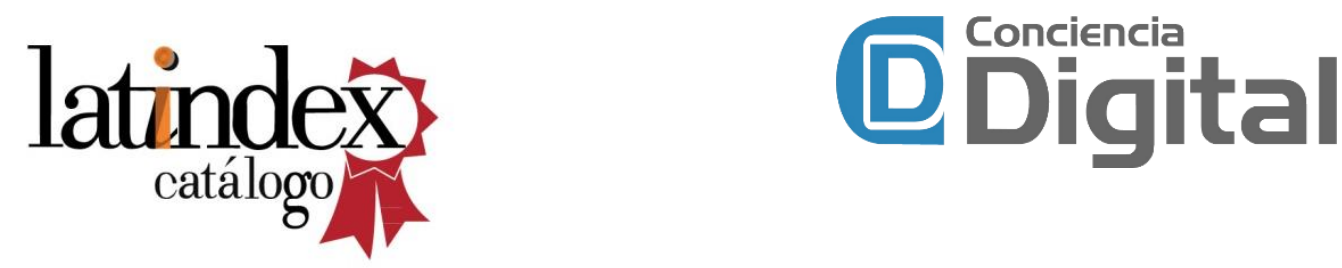

Indexaciones

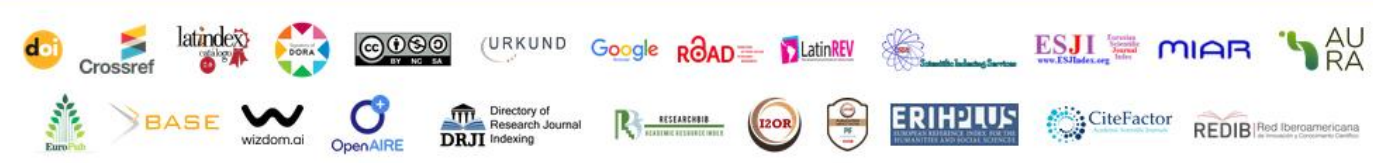

